\title{
A Volatility Decomposition Control Variate Technique for Monte Carlo Simulations of Heath Jarrow Morton Models
}

\author{
Carl Chiarella ${ }^{1}$ - Les Clewlow ${ }^{2}-$ Silvana Musti $^{3}$
}

\begin{abstract}
The aim of this work is to develop a simulation approach to the yield curve evolution in the Heath, Jarrow \& Morton (1992) framework. The stochastic quantities considered as affecting the forward rate volatility function are the spot rate and the forward rate. A decomposition of the volatility function into a Hull \& White (1990) volatility and a remainder allows us to develop an efficient Control Variate Method that makes use of the closed form solution of the Hull and White call option. This technique considerably speeds up the simulation algorithm to approximate call option values with Monte Carlo simulation. ${ }^{4}$
\end{abstract}

\section{Introduction}

The Heath, Jarrow and Morton (1992) model represents one of the most general and feasible approaches to the modelling of the term structure of interest rate in the arbitrage free environment. Heath, Jarrow and Morton proved that under no-arbitrage conditions the instantaneous forward rate process can be expressed by the stochastic integral equation

$$
f(t, T)=f(0, T)+\int_{0}^{t} \sigma^{*}(s, T, \cdot) d s+\int_{0}^{t} \sigma(s, T, \cdot) d \widetilde{W} s
$$

where:

\footnotetext{
${ }^{1}$ School of Finance and Economics, University of Technology, Sydney, Australia

${ }^{2}$ Lacima Group and University of Technology, Sydney, Australia

${ }^{3}$ Facolta' di Economia, Universita' degli Studi di Foggia, Italy

${ }^{4}$ We thank Tomas Björk and Nadima El-Hassan for their helpful comments during the development of this work. We are particularly indebted to the anonymous referee whose suggestions greatly improved the paper.
} 
$f(t, T)$ is the instantaneous forward rate at time $t$ applicable to time $T, t<T$;

$f(0, T)$ is the initial forward rate curve observable at time $t=0 ;$

$\sigma(t, T, \cdot)$ is the instantaneous forward rate volatility function;

$\sigma^{*}(s, T, \cdot) d s=\sigma(s, T, \cdot) \int_{s}^{T} \sigma(s, u, \cdot) d u ;$

$\widetilde{W}$ is the Wiener process generated by the equivalent martingale measure $\widetilde{P}$.

The third argument in the volatility function $\sigma(t, T, \cdot)$ indicates the possible dependence on other path dependent variables such as the spot rate or the forward rate itself. The case when the volatility function is independent of any path dependent quantities, and hence is a function only of the time and maturity, has been extensively analyzed. This results in the so called Gaussian HJM class of models, for which Black-Scholes type formulae are available (Brace \& Musiela (1994)). The Hull and White multifactor models (Hull \& White (1994)) also fall into this framework. Chiarella \& Kwon (2001a) discuss how a number of popular models may be formed under the HJM framework.

However volatility functions which are only functions of time and maturity do not allow sufficiently rich movements for the forward rate curve. For instance it is not possible to have volatility depending on the level of rates.

The simplest way to obtain volatility functions which are dependent on the level of rates is to allow the forward rate volatility function to depend on the instantaneous spot rate of interest $r(t)$, i.e.

$$
\sigma(t, T, \cdot)=\sigma(t, T, r(t))
$$

The volatility function (2) has the path dependent quantity $r(t)$ among its arguments. The stochastic dynamics of the forward rate and the spot rate are, generically, nonMarkovian (the entire path is necessary to capture the dynamics of the process). Jeffrey (1995) gives conditions under which the stochastic dynamics under (2) may be Markovianised with one state variable. In some special, but useful for applications, cases it is possible to transform the model into a two-dimensional state variable 
Markovian model (see Ritchken \& Sankarasubramanian (1995)). These two variables capture the information contained in the term structure so that numerical algorithms to price options have only to keep track of them. It also turns out to be possible in this framework to obtain closed form expressions for bond prices and feasible numerical schemes for the evaluation of options (Ritchken \& Sankarasubramanian (1995), Bhar \& Chiarella (1997), Chiarella \& El-Hassan (1999), Inui \& Kijima (1998) and Chiarella \& Kwon (2001b)).

Whilst the volatility function (2) certainly captures the effect of rate levels on the movement of the forward curve, it is only capturing the effect of rates at the shortest end of the yield curve. It is well known empirically that rates at different maturities exhibit different volatilities. Such effects can only be captured by allowing the volatility function to depend on the forward curve itself.

One approach to capture such dependence on the level of the forward curve is to allow the forward rate volatility function to depend on the spot interest rate $r(t)$ and on the levels the forward rate assumes at the $n$ fixed maturities:

$$
\tau_{1}, \tau_{2}, \cdots, \tau_{n} \quad t \leq \tau_{1} \leq \tau_{2} \leq \cdots \leq \tau_{n} .
$$

The volatility function then assumes the more general form:

$$
\sigma(t, T, \cdot)=\sigma\left(t, T, r(t), f\left(t, \tau_{1}\right), f\left(t, \tau_{2}\right), \cdots, f\left(t, \tau_{n}\right)\right),
$$

where $f\left(t, \tau_{i}\right)$ is the instantaneous forward rate applicable to the fixed maturity $\tau_{i}$. Chiarella \& Kwon (2001b) call these the fixed tenor forward rates and show that for particular specifications of the volatility structure it is possible to obtain an $(n+1)$ dimensional Markovian representation of the dynamics and it is also possible to obtain an explicit formula for the price of bonds. The higher dimensionality of the stochastic dynamics makes option pricing a more challenging numerical task, however some results using finite difference methods have been obtained by Bhar, Chiarella, El-Hassan \& Zheng (2000). A general theory for the existence and construction of finite dimensional Markovian realizations has been developed in Björk \& Svensson (2001) and Björk \& Landén (2002). This general theory has been extended in Filipović \& Teichmann(2001). 
The volatility function (3) can be considered as a particular case of a volatility function which depends on the entire forward curve and may be written as

$$
\sigma(t, T, \cdot)=\sigma(t, T, r(t), f(t, T)) \text {. }
$$

The major difficulty with working with volatility functions of the form (4) is that it seems impossible to reduce the stochastic dynamics to Markovian form using the techniques of Ritchken \& Sankarasubramanian (1995), Bhar \& Chiarella (1997) and Chiarella \& Kwon (2001b): Filipović \& Teichmann (2001) show that in this case the stochastic dynamics are non-Markovian in an essential way.

A functional form such as (3) is used by Amin \& Morton (1994) in their empirical study of forward rate volatility functions. However their paper does not make entirely clear how they handled many of the numerical difficulties associated with such volatility functions.

Jarrow (1996) uses binomial trees to evaluate European and American options on the zero-coupon bond in the HJM framework. The considered volatility functions are the deterministic functions:

$$
\sigma(t, T)=\sigma_{0} \quad \text { and } \quad \sigma(t, T)=\xi e^{-\eta(T-t)},
$$

and the nearly proportional volatility function:

$$
\sigma(t, T)=\eta(t, T) \min (f(t, T), M),
$$

where $\eta(t, T)$ is a deterministic function and $M$ is a positive constant. The bound $M$ is included to keep the forward rate from exploding in finite time.

The binomial tree is not recombining (therefore is called "bushy"): after $N$ time steps the number of nodes obtained is $2^{N}$ so that it results in a computationally inefficient scheme requiring a large number of time steps. However, as pointed out by Jarrow (1996), a large number of time steps is not always essential to obtain good approximations. For European options and American options bushy trees provide very accurate values already using step sizes of 10 or 12 . 
Convergence rate and accuracy of the non-recombining HJM forward rate tree are tested by Radhakrishnan (1999) in the Hull and White formulation of the HJM model: European and American bond option values are found to converge in 10-12 steps for maturities up to five years.

Carr \& Yang (1998) apply Markov Chain Approximation using Monte Carlo simulations to price European and American derivatives in an HJM framework. These authors have considered both deterministic and stochastic volatility structures. In particular (referring just to their single factor volatility specifications) among the cases of deterministic volatility (Ho \& Lee (1986), Hull \& White (1990)), the authors test the Markov Chain Approximation method by calculating European and American put option on zero-coupon bonds and comparing the obtained results respectively with available closed form solutions and the binomial tree solution. They apply Markov Chain Approximation both to deterministic volatility functions (Ho \& Lee (1986), Hull \& White (1990)) and to two different types of one factor stochastic volatility functions. The first stochastic volatility (Li, Ritchken \& Sankarasubramanian (1995)) is proportional to the spot rate $r(t)$

$$
\sigma(t, T)=\sigma_{0} e^{-\kappa(T-t)}[r(t)]^{\beta}, \quad \sigma(t)=0.1, \beta=1, \kappa=0.02
$$

The spot rate $r(t)$ is the only stochastic component in the volatility function, and the model turns out to be Markovian in two state variables. The second non deterministic volatility function is the one-factor nearly proportional HJM specification equation (5) with $M=1$ and $\eta(t, T)=0.1$. In this case the model remains non Markovian. The authors then go on to value both European and American options on zero-coupon bonds. They compare the values with the numerical results obtained with the nonrecombining trees method (European and American) and standard Monte Carlo (European only), suggesting that the method used gives an accurate valuation of European and American bond options, at least as viable as the non-recombining trees method.

In this paper we consider a fairly general case of proportional volatility models, by assuming a volatility function of the form 


$$
\sigma(t, T, \cdot)=e^{-\lambda(T-t)}\left[a_{0}+a_{r} r(t)+a_{f} f(t, T)\right]^{\gamma}, \quad \gamma>0
$$

In section 2 we outline the straightforward application of Monte Carlo simulation to the Heath Jarrow Morton model based on the Euler-Maruyama discretisation of the forward rate dynamics. In section 3 we outline the control variate technique approach to speed-up the Monte Carlo simulations. We show how a decomposition of the forward rate volatility into a (Gaussian) Hull and White component and a remainder allows us to use the known Hull and White solution for bond options to develop a very effective control variate. Section 4 concludes.

\section{The Numerical Scheme}

We consider the problem of derivative security valuation from the perspective of time $t=0$ where we have available the initial forward curve $f(0, T)$. Such valuation will requires the simulation of the evolution of the entire forward rate dynamics under the volatility specification. In this section we describe the algorithm developed and by its numerical implementation we determine zero-coupon bond prices and European call options on zero-coupon bond prices by use of Monte Carlo simulation. The algorithm is based on the Euler-Maruyama discretisation of stochastic integrals. The accuracy of the numerical results obtained is tested in the particular case of the volatility function representing the Hull and White formulation of the HJM framework.

\subsection{Scheme}

The basic task of the numerical algorithm we are using is to simulate one possible evolution of the forward rate curve over the horizon time $(0, \bar{T})$, given the initial forward curve $f(0, T), T \in[0, \bar{T}]$. We divide $(0, \bar{T})$ into $N$ intervals of length $\Delta t=\bar{T} / N$ so that $t=n \Delta t$ and $T=m \Delta t$, with $0 \leq t \leq T \leq \bar{T}$. 
We approximate the stochastic integral equation (1) according to the Euler-Maruyama scheme (Kloeden \& Platen (1992)). Hence we obtain the generic recursive scheme for the forward curve evolution:

$$
\begin{gathered}
f((n+1) \Delta t, m \Delta t)=f(n \Delta t, m \Delta t)+\sigma(n \Delta t, m \Delta t, \cdot) \sum_{i=n}^{m-1} \sigma(n \Delta t, i \Delta t) \Delta t \\
+\sigma(n \Delta t, m \Delta t, \cdot) \Delta \widetilde{W}(n+1) .
\end{gathered}
$$

This scheme is initiated by considering the values that the forward rate curve at time zero, $f(0, T)$, assumes at the extremes of each interval, i.e. $f(0,0)=r(0)$, $f(0, \Delta t), f(0,2 \Delta t), \cdots, f(0, N \Delta t)$. Musti (2001) contains more detailed discussion of the setting-up of the numerical algorithm and provides a number of implementations illustrating its numerical accuracy.

\subsection{The Initial Bond Price}

A useful way to test the accuracy of the numerical algorithm is to use it to compute the time zero value of a $T$-maturity zero-coupon bond. Knowledge of the initial forward curve $f(0, T)$ enables us to calculate exactly the value according to the formula

$$
P(0, T)=e^{-\int_{0}^{T} f(0, s) d s}
$$

On the other hand, according to the HJM model the same value can be obtained as the expectation under the equivalent martingale measure of the reciprocal of the money market account

$$
P(0, T)=\mathbb{E}_{\widetilde{Q}}\left[e^{-\int_{0}^{T} r(s) d s} \mid \mathcal{F}_{0}\right]
$$

Using the numerical procedure outlined in section 2.1 we calculate the $i$-th inverse of the money market account:

$$
P_{i}(0, \bar{T})=e^{-\sum_{j=0}^{N-1} r_{i}(j \Delta t) \Delta t}
$$


The procedure is repeated simulating $\prod$ paths and the approximate time zero bond price value, $P_{M C}(0, \bar{T})$, is calculated as

$$
P_{M C}(0, \bar{T})=\frac{1}{\prod} \sum_{i=0}^{\Pi} P_{i}(0, \bar{T}) .
$$

As an example we take the initial forward curve and the volatility function

$$
f(0, T)=0.08-0.03 e^{-0.2 T},
$$

and

$\sigma(t, T, r(t), f(t, T)):=e^{-0.2(T-t)}[0.016476-1.3353 r(t)+1.19843 f(t, T)]$.

The numerical results obtained with maturity $T=1$ are shown in Table 1 .

Comparing the numerical results obtained from approximating (8) by simulation with the analytical bond price value calculated using the formula (7), we observe how bond price results are consistent with this value already with a relatively small number of time steps: third decimal place accuracy is obtained with the discretization $N=150$ and a simulation of $N=1000$ paths, providing some evidence of the effectiveness of this numerical method.

\subsection{Zero-Coupon Bond Evaluation}

Consider the zero-coupon bond with maturity $\bar{T}$. We wish now to calculate the value that the bond will assume at the generic time $t_{0}, 0 \leq t_{0} \leq \bar{T}$, given the information available at time zero: the initial curve $f(0, T)$ and the volatility function $\sigma(t, T, r(t), f(t, T))$. The single $i$-th path of the simulation gives one possible realization of the forward rate curve at time $t_{0}, f_{i}\left(t_{0}, T\right)$, and from this curve we calculate the $i$-th bond value, $P_{i}\left(t_{0}, \bar{T}\right)$, according to

$$
P_{i}\left(t_{0}, \bar{T}\right):=e^{-\int_{t_{0}}^{\bar{T}} f_{i}\left(t_{0}, s\right) d s}
$$


The integration in equation (11) is a very simple and rapid calculation, obtained using the Euler-Maruyama approximation of the integral

$$
\int_{t_{0}}^{\bar{T}} f_{i}\left(t_{0}, s\right) d s=\sum_{i=n}^{N-1} f_{i}(n \Delta t, i \Delta t) \Delta t
$$

Formula (11) is needed to calculate the bond option payoffs required in section 2.4.

\subsection{European Options on Zero-Coupon Bonds}

Once the value of the bond can be determined at any time $t, 0 \leq t \leq \bar{T}$, European options values are derived by taking the expectation of the payoff at the option maturity time with respect to the risk-neutral measure $\widetilde{P}$. Suppose we wish to price at time 0 a call option on the zero-coupon bond with maturity $\bar{T}$. The option considered matures at time $T_{C}, 0 \leq T_{C} \leq \bar{T}$ and its exercise price is $E$. The option value is given by

$$
C\left(0, T_{C}, \bar{T}\right)=\mathbb{E}_{\widetilde{P}}\left[e^{-\int_{0}^{T_{C}} r(s) d s}\left(P\left(T_{C}, \bar{T}\right)-E\right)^{+} \mid \mathcal{F}_{0}\right]
$$

We divide the time interval $(0, \bar{T})$ into $N$ intervals of equal length $\Delta t=\frac{\bar{T}}{N}$, so that the bond and the option maturity times are respectively $T_{C}=n \Delta t$ and $T=N \Delta t$. Alternatively we may use the $T_{c}$-forward probability measure $P^{*}$ to calculate the option value according to

$$
C\left(0, T_{C}, \bar{T}\right)=P\left(0, T_{C}\right) \mathbb{E}_{P^{*}}\left[\left(P\left(T_{C}, \bar{T}\right)-E\right)^{+} \mid \mathcal{F}_{0}\right]
$$

Simulation under the $P^{*}$ measure is carried out by the replacement of Wiener increments according to

$$
d \widetilde{W}(t) \rightarrow d W^{*}(t)-\sigma_{P}\left(t, T_{C}\right) d t
$$

where $\sigma_{P}\left(t, T_{C}\right)$ is the volatility of the bond price process implied by (1) and is given by 


$$
\sigma_{P}\left(t, T_{C}\right)=-\int_{t}^{T_{C}} \sigma(t, v) d v
$$

The $T_{c}$-forward probability measure has proved useful in allowing the derivation of Black-type option pricing formulas for the class of volatility functions allowing such representations. However for the class of volatility functions requiring a simulation based approach use of one measure does not seem to offer any advantage over the other. The transformation (13) merely changes the drift terms of the stochastic differential equations being simulated. Under both measures we need to simulate the forward rate dynamics to calculate all required quantities. Here we have chosen to simulate under $\widetilde{P}$.

Thus the call option value is obtained from

$$
C_{M C}(0, n \Delta t, N \Delta t)=\frac{1}{\Pi} \sum_{i=1}^{\Pi} C_{i}(0, n \Delta t, N \Delta t),
$$

where

$$
C_{i}(0, n \Delta t, N \Delta t)=\left(P_{i}(n \Delta t, N \Delta t)-E\right)^{+} e^{-\sum_{j=0}^{N-1} r_{i}(j \Delta t) \Delta t} .
$$

and $P_{i}$ is computed by (11). Examples of numerical results are shown in Table 4: the value of the 6 months maturity call option on the 1 dollar pure discount Bond with maturity 1 year is obtained. Monte Carlo simulation with the number of paths equal to 1000 gives already a call option value with 3 decimal places accuracy. The put option value is obtained analogously.

\subsection{The Hull and White Case}

The Hull and White model is special case of the HJM model and offers the opportunity to test the numerical algorithm at least in the case of deterministic volatility function of the form

$$
\sigma(t, T)=a_{0} e^{-\lambda(T-t)}
$$

since in this case it is possible to obtain an exact expression for the European bond option price. The form (16) is obtained from equation (6) simply by setting $a_{r}=a_{f}=0$ and $\gamma=1$. 
We consider a call option with maturity 0.5 on a one year zero-coupon bond and we apply a volatility function (16) with parameters $\lambda=0.2$ and $a_{0}=0.01647$.

The initial forward curve is

$$
f(0, T)=0.08-0.03 e^{-0.2 T}
$$

and the exercise price considered is $E=0.948366$.

Table 3 shows the closed form solution value and the numerical values obtained by Monte Carlo simulation. Decreasing time steps have been chosen varying from 1/150 to $1 / 300$ of a year. The observed call option values are obtained by using different numbers of paths, as shown in the second column of Table 3. As can be observed from call option values in the third column compared with the exact call option value displayed in the second row, 3 decimal place accuracy is obtained in every simulation. In almost all the simulations using the discretization $N=150$ and $N=180$, the accuracy reaches four decimal places. Standard errors are obviously decreasing as the number of paths increases and don't appear to be influenced by the dimension of the time step. It is interesting to notice how the estimated call option value reaches three decimal places accuracy in the cases of bigger time step discretisazion (see in Table 3 the values obtained for $N=150$ and $N=180$ ).

\section{Control Variate Technique and Volatility Decomposition}

\subsection{The Control Variate Method}

The Control Variate Method is discussed at length by Clewlow \& Caverhill (1994) and Clewlow \& Strickland (1998). Here we apply it to reduce the error on the evaluation of European call options values under HJM with Monte Carlo simulation by using the closed form solution of the Hull and White call option. On each path the evolution of the forward rate curve according to both the volatility function

$$
\sigma(t, T, r(t), f(t, T))=e^{-\lambda(T-t)}\left[a_{0}+a_{r} r(t)+a_{f} f(t, T)\right]^{\gamma},
$$

and the Hull and White model volatility 


$$
\sigma(t, T)=a_{0}^{\gamma} e^{-\lambda(T-t)}
$$

is obtained. From the $i$-th path the two discounted payoffs are respectively:

$$
C_{i}^{H J M}\left(0, T_{C}, T\right)
$$

and

$$
C_{i}^{H W}\left(0, T_{C}, T\right)
$$

The values obtained using Monte Carlo simulation on the $\Pi$ paths are:

$$
C_{M C}^{H J M}\left(0, T_{C}, T\right)=\frac{1}{\Pi} \sum_{i=1}^{\Pi} C_{i}^{H J M}\left(0, T_{C}, T\right),
$$

and

$$
C_{M C}^{H W}\left(0, T_{C}, T\right)=\frac{1}{\Pi} \sum_{i=1}^{\Pi} C_{i}^{H W}\left(0, T_{C}, T\right)
$$

Indicate by $C^{H J M}$ the unknown value of the call option in case of volatility function (17), and $C^{H W}$ the analytical value of the call option in case of volatility function (18) . The control variate method seeks to reduce the error of the Monte Carlo estimation,

$$
C_{M C}^{H J M}\left(0, T_{C}, T\right)-C^{H J M},
$$

by using the known error obtained by simulating the Hull and White case on the same set of paths, namely

$$
C_{M C}^{H W}\left(0, T_{C}, T\right)-C^{H W}
$$

The option approximation adjusted by the control variate method is therefore:

$$
C_{c v}^{H J M}=C_{M C}^{H J M}-C_{M C}^{H W}+C^{H W}
$$

\subsection{The Hull and White Decomposition}

The calculation in (19) will be even more effective if we can find a way to speed up the calculation of $C_{M C}^{H J M}$ and $C_{M C}^{H W}$. In this section we show how this may be achieved by decomposing the volatility function into a Hull and White volatility component and a remainder. The advantage of this decomposition is that the Hull and White model expressed in the HJM formulation, has deterministic drift and diffusion term. 
Both the deterministic and the stochastic integral appearing in the Hull and White part of the rearranged forward rate evolution can be exactly calculated. The stochastic integral arising from the remainder component of the volatility decomposition still needs to be approximated by the Euler-Maruyama method. However it can be expected to be of an order of magnitude less than the original non-decomposed stochastic integral, and so it should contribute less to the error. The Hull and White model can be obtained considering the volatility function of the form:

$$
\sigma_{H W}(t, T)=a_{0}^{\gamma} e^{-\lambda(T-t)},
$$

so that the integral form of the forward rate stochastic process under the Hull and White model assumption is:

$$
\begin{aligned}
& f_{H W}(t, T)=f(0, T)+\int_{0}^{t} \alpha_{H W}(s, T) d s+\int_{0}^{t} \sigma_{H W}(s, T) d \widetilde{W}(s), \\
& \alpha_{H W}(s, T)=\sigma_{H W}(s, T) \int_{s}^{T} \sigma_{H W}(s, v) d v .
\end{aligned}
$$

The volatility function (17) can be rearranged to separate out the Hull and White component by writing

$$
\begin{aligned}
\sigma(t, T, \cdot) & =e^{-\lambda(T-t)}\left(a_{0}+a_{r} r(t)+a_{f} f(t, T)\right)^{\gamma} \\
& =a_{0}^{\gamma} e^{-\lambda(T-t)}+\left\{\left[a_{0}+a_{r} r(t)+a_{f} f(t, T)\right]^{\gamma}-a_{0}^{\gamma}\right\} e^{-\lambda(T-t)} \\
& =\sigma_{H W}(t, T)+\sigma_{r e m}(t, T, \cdot)
\end{aligned}
$$

where

$$
\sigma_{\text {rem }}(t, T, \cdot)=\left\{\left[a_{0}+a_{r} r(t)+a_{f} f(t, T)\right]^{\gamma}-a_{0}^{\gamma}\right\} e^{-\lambda(T-t)},
$$

and intuitively we would expect this quantity to be of smaller order of magnitude than the original volatility function. The forward rate process drift term, expressed in terms of the foregoing decomposition of the volatility function becomes:

$$
\begin{gathered}
\alpha(t, T, \cdot)=\left(\sigma_{H W}(t, T)+\sigma_{r e m}(t, T, \cdot)\right) \int_{t}^{T}\left(\sigma_{H W}(t, v)+\sigma_{r e m}(t, v, \cdot)\right) d v \\
=\sigma_{H W}(t, T) \int_{t}^{T} \sigma_{H W}(t, v) d v+\left[\sigma_{H W}(t, T)+\sigma_{r e m}(t, T, \cdot)\right] \\
\int_{t}^{T} \sigma_{r e m}(t, v, \cdot) d v+\sigma_{r e m}(t, T, \cdot) \int_{t}^{T} \sigma_{H W}(t, v),
\end{gathered}
$$

that will be expressed in concise form as

$$
\alpha(t, T, \cdot)=\alpha_{H W}(t, T)+\alpha_{r e m}(t, T, \cdot) .
$$


The forward rate stochastic integral equation assumes the form:

$$
\begin{aligned}
f(t, T)=f & (0, T)+\int_{0}^{t} \alpha_{H W}(s, T) d s+\int_{0}^{t} \sigma_{H W}(s, T) d \widetilde{W}(s) \\
& +\int_{0}^{t} \alpha_{r e m}(s, T, \cdot) d s+\int_{0}^{t} \sigma_{r e m}(s, T, \cdot) d \widetilde{W}(s) .
\end{aligned}
$$

This decomposition represents a possible improvement to the numerical implementation since two of the integrals on the right hand side of (22) can be evaluated exactly.

The first integral $\int_{0}^{t} \alpha_{H W}(s, T)$ can be analytically evaluated, since it only involves time deterministic functions.

The stochastic integral

$$
\int_{0}^{t} \sigma_{H W}(s, T) d \widetilde{W}(s)
$$

represents a Gaussian random variable with zero expected value, and whose variance can be calculated analytically.

\subsection{The Recursive Formula}

To obtain the recursive relation for the forward rate within the framework of this section, we discretize as in section 2.1. The process (22) at time $(t+\Delta t)$ is

$$
\begin{aligned}
f(t+\Delta t, T)=f & (0, T)+\int_{0}^{t+\Delta t} \alpha_{H W}(s, T) d s+\int_{0}^{t+\Delta t} \sigma_{H W}(s, T) d \widetilde{W}(s) \\
& +\int_{0}^{t+\Delta t} \alpha_{r e m}(s, T, \cdot) d s+\int_{0}^{t+\Delta t} \sigma_{r e m}(s, T, \cdot) d \widetilde{W}(s) .
\end{aligned}
$$

By subtracting (22) from (23) we obtain the relation between the forward curve at time $t$ and the forward curve at time $(t+\Delta t)$. Thus

$$
\begin{aligned}
f(t+\Delta t, T)=f & (t, T)+\int_{t}^{t+\Delta t} \alpha_{H W}(s, T) d s+\int_{t}^{t+\Delta t} \sigma_{H W}(s, T) d \widetilde{W}(s) \\
& +\int_{t}^{t+\Delta t} \alpha_{r e m}(s, T, \cdot) d s+\int_{t}^{t+\Delta t} \sigma_{r e m}(s, T, \cdot) d \widetilde{W}(s) .
\end{aligned}
$$

Consider the first integral on the right hand side of (24) 


$$
\begin{aligned}
\int_{t}^{t+\Delta t} \alpha_{H W}(s, T) d s=\int_{t}^{t+\Delta t} & \left(\sigma_{H W}(s, T) \int_{s}^{T} \sigma_{H W}(s, v) d v\right) d s= \\
& \int_{t}^{t+\Delta t}\left(a_{0}^{\gamma} e^{-\lambda(T-s)} \int_{s}^{T} a_{0}^{\gamma} e^{-\lambda(v-s)} d v\right) d s .
\end{aligned}
$$

This integral can be exactly calculated. The inside integral is:

$$
\int_{s}^{T} a_{0}^{\gamma} e^{-\lambda(v-s)} d v=-\frac{1}{\lambda} a_{0}^{\gamma}\left[e^{\lambda(s-T)}-1\right]=: h(s, T),
$$

and, substituting (26) into (25) we obtain:

$$
\begin{aligned}
\int_{t}^{t+\Delta t} \alpha_{H W}(s, T) d s & =-\frac{a_{0}^{2 \gamma}}{\lambda} \int_{t}^{t+\Delta t}\left(e^{-2 \lambda(T-s)}-e^{-\lambda(T-s)}\right) d s \\
& =-\frac{a_{0}^{2 \gamma}}{2 \lambda^{2}}\left(e^{-2 \lambda(T-(t+\Delta t))}-2 e^{-\lambda(T-(t+\Delta t))}-e^{-2 \lambda(T-t)}+2 e^{-\lambda(T-t)}\right) \\
& =: g(t, T, \Delta t) .
\end{aligned}
$$

The stochastic integral in the Hull and White part is the normally distributed random variable

$$
\widetilde{X}:=\int_{t}^{t+\Delta t} \sigma_{H W}(s, T) d \widetilde{W}(s)
$$

We are calculating the values from the perspective of the information set at time zero, so conditioning to time zero we readily calculate that

$$
E_{0}[\widetilde{X}]=0
$$

and

$$
\operatorname{Var}_{0}[\widetilde{X}]=\frac{a_{0}^{2 \gamma}}{2 \lambda}\left[e^{-2 \lambda(T-(t+\Delta t))}-e^{-2 \lambda(T-t)}\right]=: v(t, t+\Delta t, T)
$$

Thus

$$
\widetilde{X}=\sqrt{v(t, t+\Delta t, T)} \widetilde{\xi}_{t}
$$

where $\tilde{\xi}$ is the standard Normal random variable.

Equation (24) becomes 


$$
\begin{aligned}
f(t+\Delta t, T)=f & (t, T)+g(t, t+\Delta t, T)+\sqrt{v(t, t+\Delta t, T)} \xi_{t} \\
& +\int_{t}^{t+\Delta t} \alpha_{r e m}(s, T, \cdot) d s+\int_{t}^{t+\Delta t} \sigma_{r e m}(s, T, \cdot) d \widetilde{W}(s) .
\end{aligned}
$$

Using the discretization outlined in section 2 the $(n+1) \Delta t$ time forward curve is obtained from the curve at the immediate former step, $n \Delta t$ time forward curve. In particular, considering as general maturity the $T=m \Delta t$, we have:

$$
\begin{aligned}
f((n+1) \Delta t, m \Delta t)= & f(n \Delta t, m \Delta t)+g(n \Delta t, \Delta t, m \Delta t) \\
& +\sqrt{v(n \Delta t, \Delta t, m \Delta t)} \xi_{n+1}+\alpha_{r e m}(n \Delta t, m \Delta t, \cdot) \\
& +\sigma_{r e m}(n \Delta t, m \Delta t, \cdot) \xi_{n+1} \sqrt{\Delta t}
\end{aligned}
$$

where we approximate $\alpha_{r e m}(n \Delta t, m \Delta t, \cdot)$ according to

$$
\begin{aligned}
\alpha_{r e m}(n \Delta t, m \Delta t, \cdot) & =\left[\sigma_{H W}(n \Delta t, m \Delta t)+\sigma_{r e m}(n \Delta t, m \Delta t, \cdot)\right] . \\
& \sum_{i=n}^{m-1} \sigma_{r e m}(n \Delta t, i \Delta t, \cdot) \Delta t+\sigma_{r e m}(n \Delta t, m \Delta t, \cdot) h(n \Delta t, m \Delta t) .
\end{aligned}
$$

Using the recursive formula (28) we calculate $C_{M C}^{H J M}$ and $C_{M C}^{H W}$ (with $a_{r}=a_{f}=0$ ) according to (15).

\subsection{Numerical Results}

The control variate adjusted results are shown in Table 4. The standard errors of the values calculated with the control variate method are of the order of one third with respect the solution obtained previously. This reduction seems fairly uniform across the number of paths and order of discretisation. It signifies that using the control variate method one may obtain the same order of accuracy (with respect to standard Monte Carlo simulations) with one ninth the number of paths. These results indicate that the control variate technique is very effective for this class of HJM model in speeding up the simulations and can yield good results in Monte Carlo simulations even with a relatively small number of paths.

\section{Conclusions}


In this paper we have considered the Heath-Jarrow-Morton model in the case when the forward rate volatility function depends not only on time to maturity and the instantaneous spot rate of interest (the most common situations discussed in the literature), but also on the entire forward rate curve. In this case it seems that we are forced to deal with a stochastic integral equation for the forward rate which is nonMarkovian in an essential way so that its reduction to Markovian form by the trick of a finite expansion of the state space does not seem possible. Thus numerical calculations with the HJM model in this form need to be undertaken with nonrecombining trees or by the use of Monte Carlo simulations. This paper has investigated and implemented the latter approach. A Monte Carlo scheme for simulating the evolution of the entire forward curve has been developed based on the Euler-Maruyama discretisation of stochastic integrals. Bond prices and European call options on zero-coupon bond prices have been calculated and the results using the scheme give good agreement in the case for which we have a closed form solution for the derivative prices. The main contribution of the paper has been to decompose the forward rate volatility function in a way that allows advantage to be taken of known analytical results for the volatility function corresponding to the Hull and White model. The effect of this decomposition is to reduce the numerical size of the integral that must be approximated by Monte Carlo simulations. The decomposition allows us to develop an effective application of the control variate method to increase the efficiency of the Monte Carlo simulations of European bond call option values. For the numerical examples considered here, a reduction in the standard errors of the order of one third has been obtained. This means a reduction of one ninth (compared to standard Monte Carlo) in the number of paths required to attain a given accuracy.

Future research could proceed in a number of directions.

First, investigate ways to speed up the computational time of the algorithm such as better approximation to the stochastic integrals (e.g. use of higher order methods) and use of quasi random numbers such as Fauré sequences in the Monte Carlo simulations.

Second, develop the algorithm further to evaluate American bond options and make comparison with results of Carr \& Yang (1998). Monte Carlo simulation has been 
developed as a flexible method to calculate American options written on common stock (see e.g. Barraquand \& Martineu (1995)), and so should be feasible in the context of the HJM model as studied in this paper.

Finally, perform calibration type empirical studies to "back-out" forms of the forward rate volatility function from option prices calculated using the algorithms developed here. This would complement and extend the study of Amin \& Morton (1994).

\section{Appendix:}

TABLE 1. Time Zero Bond Values

\begin{tabular}{|c|c|c|c|}
\hline \multicolumn{4}{|c|}{$P(0,1)=e^{-\int_{0}^{1} f(0, s) d s}=0.94856058$} \\
\hline$N$ & $\Pi$ & $P(0,1)$ & St.Err. \\
\hline \multirow{3}{*}{150} & 1,000 & 0.948301 & 0.000201 \\
\cline { 2 - 4 } & 5,000 & 0.948235 & 0.000087 \\
\cline { 2 - 4 } & 10,000 & 0.948260 & 0.000062 \\
\hline \multirow{3}{*}{180} & 1000 & 0.948356 & 0.000205 \\
\cline { 2 - 4 } & 5,000 & 0.948268 & 0.000087 \\
\cline { 2 - 4 } & 10,000 & 0.948365 & 0.000061 \\
\hline \multirow{3}{*}{210} & 1,000 & 0.948368 & 0.000196 \\
\cline { 2 - 4 } & 5,000 & 0.948390 & 0.000087 \\
\cline { 2 - 4 } & 10,000 & 0.948334 & 0.000062 \\
\hline \multirow{3}{*}{240} & 1,000 & 0.948003 & 0.000202 \\
\cline { 2 - 4 } & 5,000 & 0.948249 & 0.000086 \\
\cline { 2 - 4 } & 10,000 & 0.948307 & 0.000062 \\
\hline \multirow{3}{*}{270} & 1,000 & 0.948514 & 0.00019 \\
\cline { 2 - 4 } & 5,000 & 0.948378 & 0.000085 \\
\cline { 2 - 4 } 300 & 10,000 & 0.948336 & 0.000062 \\
\hline \multirow{3}{*}{} & 1,000 & 0.948502 & 0.000199 \\
\cline { 2 - 4 } & 5,000 & 0.948404 & 0.000087 \\
\cline { 2 - 4 } & 10,000 & 0.948366 & 0.000061 \\
\hline
\end{tabular}


TABLE 2

\begin{tabular}{|c|c|c|c|}
\hline \multicolumn{4}{|c|}{ Call Option Value $(E=0.948366)$} \\
\hline$N$ & $\Pi$ & $C_{M C}(0,0.5,1)$ & St.Err. \\
\hline \multirow{3}{*}{150} & 1,000 & 0.024150 & 0.000121 \\
\hline & 5,000 & 0.024232 & 0.000055 \\
\hline & 10,000 & 0.024243 & 0.000039 \\
\hline \multirow{3}{*}{180} & 1000 & 0.024283 & 0.000122 \\
\hline & 5,000 & 0.024268 & 0.000056 \\
\hline & 10,000 & 0.024242 & 0.000039 \\
\hline \multirow{3}{*}{210} & 1,000 & 0.024073 & 0.000126 \\
\hline & 5,000 & 0.024247 & 0.000055 \\
\hline & 10,000 & 0.024332 & 0.000039 \\
\hline \multirow{3}{*}{240} & 1,000 & 0.024455 & 0.000125 \\
\hline & 5,000 & 0.024298 & 0.000056 \\
\hline & 10,000 & 0.024279 & 0.000040 \\
\hline \multirow{3}{*}{270} & 1,000 & 0.024284 & 0.000119 \\
\hline & 5,000 & 0.024327 & 0.000057 \\
\hline & 10,000 & 0.024298 & 0.000039 \\
\hline \multirow{3}{*}{300} & 1,000 & 0.024327 & 0.000125 \\
\hline & 5,000 & 0.024236 & 0.000055 \\
\hline & 10,000 & 0.024312 & 0.000039 \\
\hline
\end{tabular}


TABLE 3

\begin{tabular}{|c|c|c|c|}
\hline "Col & angrican & HI & \\
\hline$C_{H V}$ & $(0,0.5$, & $=0.02428056$ & 143637 \\
\hline$N$ & $\Pi$ & $C_{M C}(0,0.5,1)$ & St.Err. \\
\hline & 1,000 & 0.024093 & 0.000156 \\
\hline 150 & 5,000 & 0.024219 & 0.000070 \\
\hline & 10,000 & 0.024229 & 0.000050 \\
\hline & 100,000 & 6 & 0.000016 \\
\hline & 1000 & 67 & 000157 \\
\hline 180 & 5,000 & 0.024259 & 0.000072 \\
\hline & 10,000 & 0.024224 & 0.000050 \\
\hline & 100,000 & 0.024311 & 0.000016 \\
\hline & 1,000 & 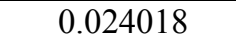 & 0.000161 \\
\hline 210 & 5,000 & 0.024240 & 0.000070 \\
\hline & 10,000 & 0.024345 & 0.000050 \\
\hline & 100,000 & 0.0242 & 0.000016 \\
\hline & 1,000 & 0.024495 & 0.000161 \\
\hline 240 & 5,000 & 0.024300 & 0.000071 \\
\hline & 10,000 & 0.024281 & 0.000051 \\
\hline & 100,000 & 0.024320 & 0.000016 \\
\hline & 1,000 & 0.024293 & 0.000153 \\
\hline 270 & 5,000 & 0.024349 & 0.000073 \\
\hline & 10,000 & 0.024304 & 0.000050 \\
\hline & 100,000 & 0.024264 & 0.000016 \\
\hline & 1,000 & 0.024346 & 0.000160 \\
\hline 300 & 5,000 & 0.024231 & 0.000071 \\
\hline & 10,000 & 0.024313 & 0.000050 \\
\hline & 100,000 & 0.024276 & 0.000016 \\
\hline
\end{tabular}


TABLE 4. Control Variate Method

\begin{tabular}{|c|c|c|c|c|c|}
\hline \multicolumn{7}{|c|}{ Call Option Value $(E=0.9483661785165)$} \\
\hline \multirow{2}{*}{$N$} & $\Pi$ & $C_{H J M}(0,0.5,1)$ & St.Err. $_{H J M}$ & $C_{c v}(0,0.5,1)$ & St.Err ${ }_{c v}$ \\
\hline \multirow{3}{*}{150} & 1,000 & 0.024153794 & 0.000119732 & 0.024341100 & 0.000039032 \\
\cline { 2 - 6 } & 5,000 & 0.024282657 & 0.000053643 & 0.024288600 & 0.000017365 \\
\cline { 2 - 6 } & 10,000 & 0.024215119 & 0.000038670 & 0.024306400 & 0.000012376 \\
\hline \multirow{3}{*}{180} & 1000 & 0.024256974 & 0.000121689 & 0.024291200 & 0.000038951 \\
\cline { 2 - 6 } & 5,000 & 0.024246360 & 0.000054358 & 0.024292400 & 0.000017405 \\
\cline { 2 - 6 } & 10,000 & 0.024319068 & 0.000038796 & 0.024273500 & 0.000012431 \\
\hline \multirow{3}{*}{210} & 1,000 & 0.024382244 & 0.000117543 & 0.024262700 & 0.000038648 \\
\cline { 2 - 6 } & 5,000 & 0.024339704 & 0.000055247 & 0.024269200 & 0.000017888 \\
\cline { 2 - 6 } & 10,000 & 0.024266608 & 0.000038989 & 0.024283000 & 0.000012487 \\
\hline \multirow{3}{*}{240} & 1,000 & 0.024281831 & 0.000118777 & 0.024301900 & 0.000038459 \\
\cline { 2 - 6 } & 5,000 & 0.024327193 & 0.000054847 & 0.024274900 & 0.000017598 \\
\cline { 2 - 6 } & 10,000 & 0.024319009 & 0.000038739 & 0.024273200 & 0.000012517 \\
\hline \multirow{3}{*}{270} & 1,000 & 0.024016787 & 0.000121956 & 0.024369100 & 0.000039667 \\
\cline { 2 - 6 } & 5,000 & 0.024257994 & 0.000054527 & 0.024298800 & 0.000017517 \\
\cline { 2 - 6 } & 10,000 & 0.024364657 & 0.000039091 & 0.024260900 & 0.000012553 \\
\hline \multirow{3}{*}{300} & 1,000 & 0.024347426 & 0.000123855 & 0.024244800 & 0.000038467 \\
\cline { 2 - 6 } & 5,000 & 0.024422593 & 0.000055999 & 0.024242700 & 0.000017981 \\
\cline { 2 - 6 } & 10,000 & 0.024319471 & 0.000038978 & 0.024268600 & 0.000012450 \\
\hline
\end{tabular}




\section{References:}

Amin, K. \& Morton, A. (1994), "Implied Volatility Functions in Arbitrage-free Term Structure Models", Journal of Financial Economics 35, 141-180.

Barraquand, J. \& Martineu, D. (1995), "Numerical Valuation of High Dimensional Multivariate American Securities", Journal of Financial and Quantitative Analysis 30(3), 383-405.

Bhar, R. \& Chiarella, C. (1997), “Transformation of Heath-Jarrow-Morton Models to Markovian Systems", European Journal of Finance 3, 1-26.

Bhar, R., Chiarella, C., El-Hassan, N. \& Zheng, X. (2000), “The Reduction of Forward Rate Dependent Volatility HJM Models to Markovian Form: Pricing European Bond Options", Journal of Computational Finance 3, 47-72.

Björk, T. \& Landén, C. (2002), "On the Construction of Finite Dimensional Realizations for Nonlinear Forward Rate Models", Finance and Stochastics 6,303-331.

Björk, T. \& Svensson, L. (2001), “On the Existence of Finite Dimensional Realizations for Nonlinear Forward Rate Models", Mathematical Finance 11(2), 205-243.

Brace, A. \& Musiela, M. (1994), “A Multifactor Gauss Markov Implementation of Heath, Jarrow and Morton", Mathematical Finance 4(3), 259-283.

Carr, P. \& Yang, G. (1998), "Simulating American Bond Options in an HJM Framework”. presented at Risk Conference, New York, September 1998.

Chiarella, C. \& El-Hassan, N. (1999), "Pricing American Bond Options in a HeathJarrow-Morton Framework". Research Paper 12,Quantitative Finance Research Group, School of Finance and Economics, University of Technology Sydney.

Chiarella, C. \& Kwon, O. (2001a), "Classes of Interest Rate Models under the HJM Framework", Asia-Pacific Financial Markets 8, 1-22.

Chiarella, C. \& Kwon, O. (2001b), "Forward Rate Dependent Markovian Transformations of the Heath-Jarrow-Morton Term Structure Model", Finance and Stochastics 5,237-257.

Clewlow, L. \& Caverhill, A. (1994), "On the Simulation of Contingent Claims", The Journal of Derivatives pp. 66-74. Fall.

Clewlow, L. \& Strickland, C. (1998), Implementing Derivative Models, John Wiley, London. 
Filipović, D. \& Teichmann, J. (2001), “Existence of Finite Dimensional Realizations for Stochastic Equations". forthcoming in Journal of Functional Analysis.

Heath, D., Jarrow, R. \& Morton, A. (1992), "Bond Pricing and the Term Structure of Interest Rates: A New Methodology for Contingent Claim Valuations", Econometrica 60(1), 77-105.

Ho, T. \& Lee, S. (1986), “Term Structure Movements and Pricing Interest Rate Contingent Claims", Journal of Finance 41(5), 1011-1029.

Hull, J. \& White, A. (1990), "Pricing Interest Rate Derivative Securities", Review of Financial Studies 3, 573-592.

Hull, J. \& White, A. (1994), "Numerical Procedures for Implementing Term Structure Models II: Two-Factor Models", The Journal of Derivatives 2, 37-48.

Inui,. K. \& Kijima, M. (1998), “A Markovian framework in multi-factor HeathJarrow-Morton Models", Journal of Financial and Quantitative Analysis 33, 423440.

Jarrow, R. (1996), Modelling Fixed Income Securities and Interest Rate Options, McGraw-Hill.

Jeffrey, A. (1995), "Single Factor Heath-Jarrow-Morton Term Structure Models Based on Spot Interest Rate Dynamics", Journal of Financial and Quantitative Analysis 30, 619-642.

Kloeden, P. \& Platen, E. (1992), Numerical Solution of Stochastic Differential Equations, Springer-Verlag.

Li, A., Ritchken, P. \& Sankarasubramanian, L. (1995), "Lattice Models for Pricing American Interest Rate Claims", Journal of Finance 50, 719-737.

Musti, S. (2001), "Numerical Investigations of the Heath Jarrow Morton Model with Forward Rate Dependent Volatility". Master of Business Thesis, School of Finance and Economics, UTS, Sydney.

Radhakrishnan, A. (1999), “An Empirical Study of the Convergence Properties of the Non-recombining HJM Forward Rate Tree in Pricing Interest Rate Derivatives". Ph.D. Thesis, Department of Finance, Stern School of Business, New York University.

Ritchken, P. \& Sankarasubramanian, L. (1995), "Volatility Structures of Forward Rates and the Dynamics of the Term Structure", Mathematical Finance 5(1), 5572. 